\title{
Clinico Mycological Study of Onychomycosis in Tertiary Care Centre of Maharashtra, India
}

\author{
Smita Kulkarni ${ }^{1}$, Jayshree Bhakre ${ }^{2 *}$ and A.S. Damle ${ }^{2}$ \\ ${ }^{1}$ Department of Microbiology, MGM Medical College, Aurangabad, Maharashtra, India \\ ${ }^{2}$ Department of Microbiology, Government Medical College, Aurangabad, Maharashtra, India \\ *Corresponding author
}

\author{
A B S T R A C T
}

Keywords

Onychomycosis, Fungal infections,

Dermatophytes, Moulds.

Article Info

Accepted:

20 September 2017

Available Online:

10 November 2017
Fungal infections of skin and its appendages are more prevalent in India due to favourable climatic conditions like temperature and humidity. Onychomycosis is chronic fungal infection of fingernails and toenails. A variety of fungi cause onychomycosis. Dermatophytes are predominant pathogens but yeasts and non dermatophyte moulds may also be implicated. Due to importance of high prevalence rate of onychomycosis this study was conducted. In the present study 100 patients suspected of onychomycosis were examined. Diagnosis of onychomycosis was based on the patient's history, physical examination, microscopy and culture of nail specimens. Higher incidence was noted in males (71\%) than females (29\%). Distal lateral subungual onychomycosis (DLSO) was the commonest clinical pattern found in $69 \%$ of patients followed by total dystrophic onychomycosis (TDO) found in $17 \%$ cases. Direct microscopy of the nail clippings in $20 \%$ $\mathrm{KOH}$ solution was positive in $61 \%$ and culture was positive in $54 \%$ cases. The common etiological agent was dermatophytes ( $79.6 \%$ cases) followed by non dermatophyte moulds ( $11.1 \%$ cases) and yeasts (9.2\% cases). Although onychomycosis is not life threatening, it can cause a significant negative impact on the quality of life of infected patients.

\section{Introduction}

Onychomycosis is fungal infection of the nails that causes discoloration, thickening, and separation from the nail bed. The importance of onychomycosis is often underestimated. Far more than being a simple cosmetic problem, infected nails serve as a chronic reservoir of infection which can give rise to repeated fungal infections of the skin.

Onychomycosis accounts for up to $50 \%$ of all nail disorders and $30 \%$ of all superficial fungal infections (Kaur et al., 2008). Prevalence rates for onychomycosis varying from $3 \%$ to $5 \%$ have been found in most studies; however, a few reports suggest a higher prevalence even up to $26 \%$ in general population (Chander Grover et al., 2012). Men are affected more frequently possibly due to frequent nail damage from sports and leisure activities (Kaur et al., 2008). Causative pathogens of onychomycosis include dermatophytes, non dermatophyte moulds and yeasts. Dermatophytes account for most $(90 \%)$ cases of onychomycosis of toenails and at least 50\% cases of fingernail infection (Ellis et al., 1997). Non dermatophyte moulds account for 1.5-6\% cases of onychomycosis (Greer et al., 1995). 
Previously regarded as contaminants, yeasts are now recognized as pathogens in fingernail infections (Vinod et al., 2000). Correct and prompt diagnosis of onychomycosis before instituting an antifungal therapy is necessary. A false negative diagnosis may lead to continued disfigurement and discomfort as well as repeated fungal infections elsewhere on the body. A false positive diagnosis may lead to unnecessary, long and expensive treatment.

As various aspects of onychomycosis are likely to differ markedly in different regions, the present study was conducted with object to isolate the causative pathogens, to determine the contributing factors and to determine various clinical patterns of onychomycosis in our region.

\section{Materials and Methods}

This cross - sectional observational study was conducted after the approval from Ethical Committee. The present study was carried out in the Department of Microbiology, Government Medical College, Aurangabad over the period of one and half year from January 2011 to July 2012. A detailed history and clinical examination of 100 suspected patients of onychomycosis were recorded.

Clinically the disease was classified as Distal and Lateral Subungual Onychomycosis (Figure1), Proximal Subungual Onychomycosis (Figure 2), White Superficial Onychomycosis and Total Dystrophic Onychomycosis (Figure 3).

The most severely affected nail was thoroughly cleaned with $70 \%$ alcohol and nail clipping/scrapping was collected. A part of this sample was dissolved in $20 \%$ potassium hydroxide $(\mathrm{KOH})$ and examined under the light microscope for fungal elements. The remaining specimen was inoculated on two sets of media (1) Sabouraud's dextrose agar (SDA) supplemented with cycloheximide $(100 \mathrm{ug} / \mathrm{ml})$ and chloramphenicol $(50 \mathrm{ug} / \mathrm{ml})$ (2) SDA without cycloheximide supplement. Both these culture tubes were incubated at $37^{\circ} \mathrm{C}$ and $25^{\circ} \mathrm{C}$ respectively for four to six weeks. Dermatophyte test medium was also used for primary isolation. The culture slopes showing fungal growth were examined for colony characters like texture of colony, surface of colony, colour of colony and pigment production.

Gram staining and germ tube test were performed on smooth moist and pasty colonies. Carbohydrate fermentation and assimilation tests were also performed to identify yeasts. Lactophenol cotton blue preparation was done on mycelial growth. Trchophyton rubrum and Trichophyton mentagrophytes were confirmed by different special tests such as urease test, hair perforation test and test for pigment enhancement on potato dextrose agar.

Because of difficulty in differentiation of pathogens from contaminants the criteria used for the diagnosis of non dermatophyte moulds were demonstration of fungal elements on $\mathrm{KOH}$ mount and isolation of non dermatophytes in pure culture on three successive occasions.

\section{Results and Discussion}

Out of the 100 cases we studied, 71 were males and 29 were females. The male to female ratio was 2.04:1. The predominant age group in males and females was 31 to 40 years (Table 1).

Involvement of fingernails was seen in 58\% of patients while toenails were involved in $33 \%$ patients. Both fingernails and toenails were involved in nine patients. In majority of patients more than one nail was involved. 
The most common clinical presentation in our study was Distal and Lateral Subungual Onychomycosis which was seen in 69 cases. Total Dystrophic Onychomycosis was seen in 17 cases, Proximal Subungual Onychomycosis in 8 cases, candidial paronychia in 5 cases and White Superficial Onychomycosis in only one case (Diagram 1).

In our study the most common factor predisposing to onychomycosis was trauma to the nails. It was found in 22 patients. In 18 patients we found history of wearing occlusive foot wears. Other predisposing factors were excessive sweating, diabetes, malnutrition and peripheral vascular diseases. However in 42 patients no associated factors were observed (Table 3 ).

In this study we found that majority of (23\%) patients were agricultural workers followed by housewives $(21 \%)$.

The 61 samples were found $\mathrm{KOH}$ positive, while the remaining 39 were found negative. Out of 100 cases 54 cases could be diagnosed by culture, thus $\mathrm{KOH}$ mount found more sensitive than culture. 46 samples were found positive by both $\mathrm{KOH}$ and culture while 31 samples were negative for both $\mathrm{KOH}$ and culture. 15 samples were positive by $\mathrm{KOH}$ alone 8 samples were positive by culture alone (Table 4).

Most common pathogenic fungus in our study was $T$. rubrum. The other dermatophytes were $T$. mentagrophytes, $T$. verrucosum, $T$. schoenlenii and T. tonsurans. We isolated candida albicans in three cases and candida krusei in two patients. Non dermatophyte moulds isolated in this study were Aspergillus niger, Aspergillus terreus, Fusarium oxysporum, Scopulariopsis brevicaulis and Scedosporium specie (Table 5).

Onychomycosis is chronic fungal infection of fingernails and toenails. There has been a recent increase in the incidence of onychomycosis. This can be attributed to various factors like, expanding number of immunocompromised patients and life style changes.

\section{Diagram.1}

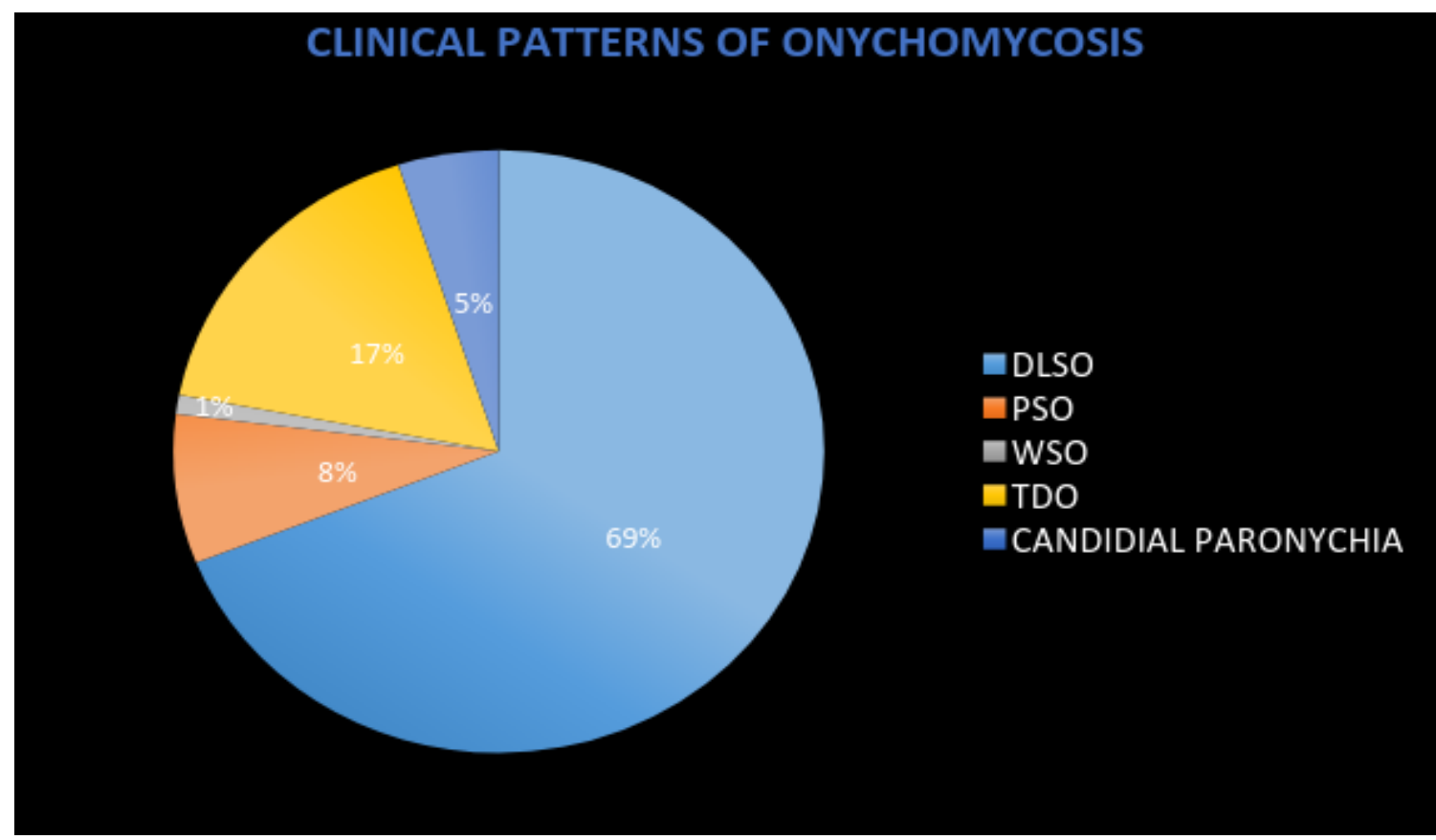


Fig.1 Distal subungual onychomycosis of great toe

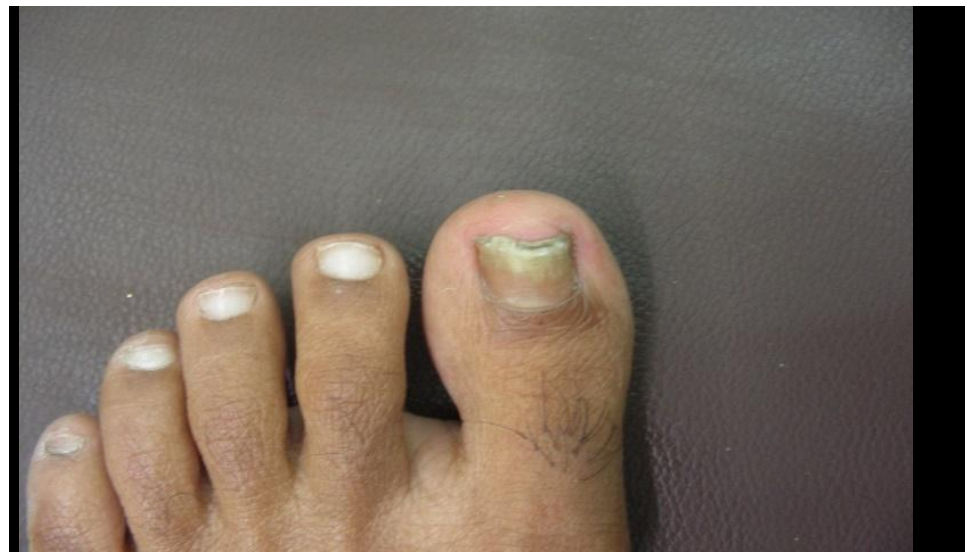

Fig.2 Proximal subungual onychomycosis of ring finger

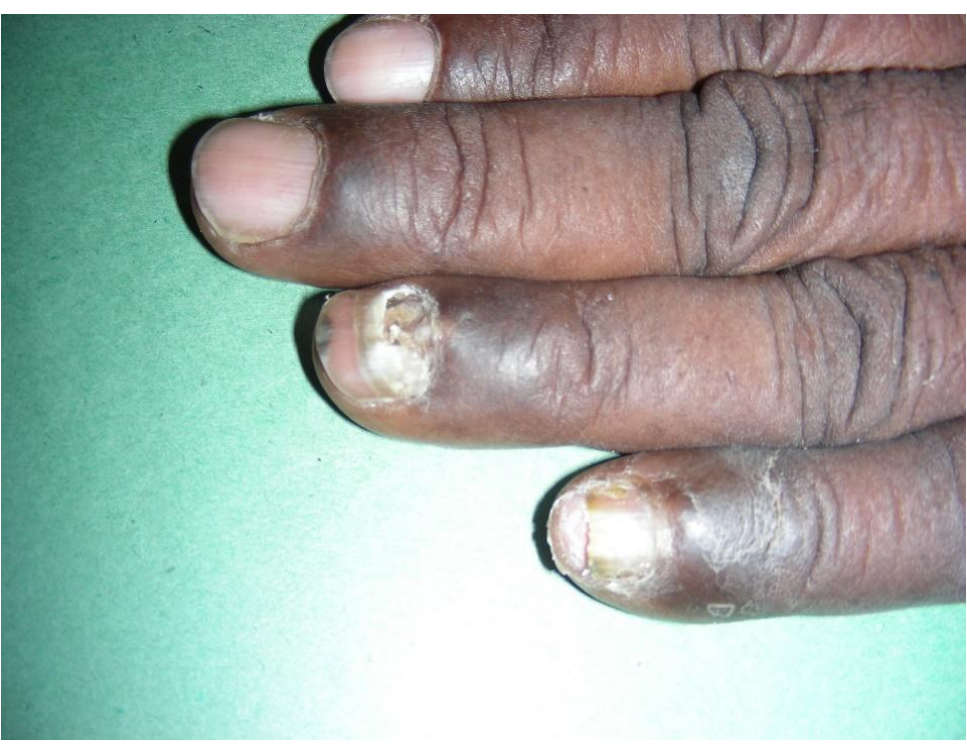

Fig.3 Total dystrophic onychomycosis of middle finger

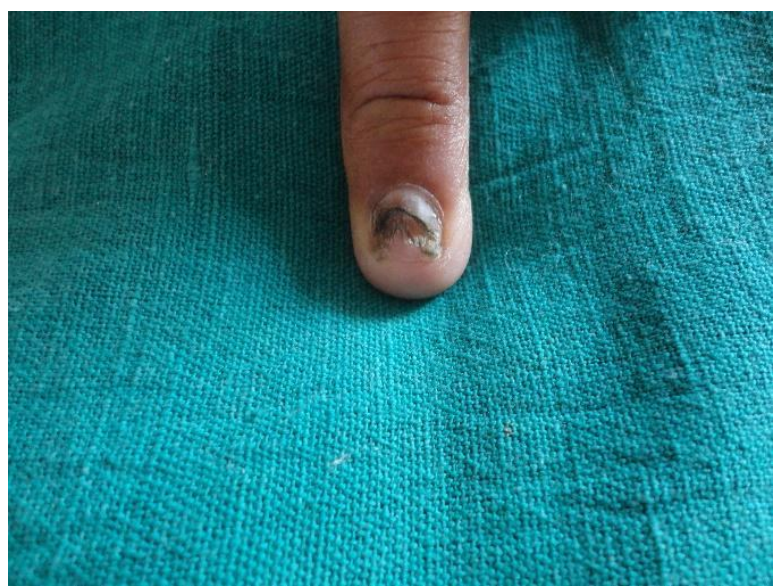


Table.1 Age and sex distribution of patients

\begin{tabular}{|c|c|c|c|}
\hline Age groups (years) & Male & Female & Total \\
\hline $0-10$ & 0 & 01 & 01 \\
\hline $11-20$ & 04 & 02 & 06 \\
\hline $21-30$ & 13 & 02 & 15 \\
\hline $31-40$ & 23 & 12 & 35 \\
\hline $41-50$ & 12 & 07 & 19 \\
\hline Above 50 years & 19 & 05 & 24 \\
\hline Total & 71 & 29 & 100 \\
\hline
\end{tabular}

Table.2 Nail changes seen in patients

\begin{tabular}{|c|c|}
\hline Nail changes & No of patients \\
\hline Discoloration & 31 \\
\hline Subungual hyperkeratosis & 24 \\
\hline Dystrophy & 23 \\
\hline Onycholysis & 11 \\
\hline Leukonychia & 06 \\
\hline Paronychia & 05 \\
\hline Total & 100 \\
\hline
\end{tabular}

Table.3 Predisposing factors of onychomycosis

\begin{tabular}{|c|c|}
\hline Predisposing factors & No of patients \\
\hline Trauma & 22 \\
\hline Occlusive footwears & 18 \\
\hline Excessive sweating & 10 \\
\hline Diabetes & 04 \\
\hline Malnutrition & 03 \\
\hline Peripheral vascular diseases & 01 \\
\hline No predisposing factor & 42 \\
\hline
\end{tabular}

Table.4 Microscopy and culture positivity of specimens

\begin{tabular}{|c|c|c|c|}
\hline & KOH positive & KOH negative & Total \\
\hline Culture positive & 46 & 08 & 54 \\
\hline Culture negative & 15 & 31 & 46 \\
\hline Total & 61 & 39 & 100 \\
\hline
\end{tabular}


Table.5 Details of fungal species isolated

\begin{tabular}{|l|c|c|}
\hline \multicolumn{1}{|c|}{ Isolated fungal species } & Number of isolates & Percentage (\%) \\
\hline T. rubrum & 26 & 48.14 \\
\hline T. mentagrophytes & 12 & 22.22 \\
\hline T. verrucosum & 03 & 5.55 \\
\hline T. scoenleinii & 01 & 1.85 \\
\hline T. tonsurans & 01 & 1.85 \\
\hline Fusarium oxysporum & 02 & 3.70 \\
\hline Aspergillus niger & 01 & 1.85 \\
\hline Aspergillus terreus & 01 & 1.85 \\
\hline Scopulariopsis brevicaulis & 01 & 1.85 \\
\hline Scedosporium species & 01 & 1.85 \\
\hline Candida albicans & 03 & 5.55 \\
\hline Candida krusei & 02 & 3.70 \\
\hline Total & 54 & 100 \\
\hline
\end{tabular}

Clinically suspected 100 cases of onychomycosis were included in this study. All the age groups and both the sexes were included in the study.

Our study revealed that incidence of onychomycosis is increasing with advancing age and it was found to be commonest in the age group 31-40 years. In this study $50 \%$ of patients were between 20-40 years, 24\% patients were above 50 years of age and only $1 \%$ below 10 years of age. High prevalence $(58.8 \%)$ of onychomycosis during the third decade has also been found in another study conducted by Adhikari et al., (2009).

Out of the 100 cases of onychomycosis in this study, $71 \%$ were males and $29 \%$ were females. Pradnya Veer et al., (2007) also reported higher $(65 \%)$ prevalence of onychomycosis among males. In contrast Jorge Lopes et al., (1999) and Bokhari et al., (1999) reported higher prevalence among females.

Onychomycosis was found to be commonest in agricultural workers (23\%), followed by housewives $(21 \%)$ and labourers (12\%). Gupta et al., (2009) also reported higher prevalence (20\%) of onychomycosis among farmers. Many patients in our study were from the occupations which involve the work that predisposes to trauma and injuries. We found that trauma to the nails was the commonest predisposing factor $(22 \%)$. The importance of trauma as predisposing factor in onychomycosis is well established (Table 2).

In present study we found more cases of fingernail onychomycosis than toenails. Various authors have reported high incidence of onychomycosis in fingernails (Pradnya Veer et al., 2007; Rigopuoulos et al., 1998). Multiple nail involvement was seen in majority of patients.

Dermatophytes were the primary pathogens $(79.6 \%)$ isolated in this study which is comparable with various studies (Pradnya Veer et al., 2007; Banerjee et al., 1989). In contrast Rigopoulos et al., (1998) and Madhuri et al., (2002) reported higher isolation rate of candida species in their study. Trichophyton rubrum (48.1\%) remains the most common pathogenic fungus isolated in our study, in accordance with other studies (Vinod S at al, 2000; Banerjee et al., 1989). 
Puri D. K et al., (1998) reported Trichophyton mentagrophytes $(50 \%)$ as the most common isolate followed by Trichophyton rubrum. Candida albicans is reported as the commonest cause of paronychial onychomycosis. This is reflected in our study where three paronychial cases showed Candida albicans on culture. Previously regarded as contaminant, yeast is now increasingly recognized as pathogen in fingernail onychomycosis (Vinod $\mathrm{S}$ et al., 2000). Non dermatophyte moulds constituted $11.1 \%$ of the total cases in this study, suggesting that they are emerging as important etiological agents in onychomycosis. Ramani et al., (1993) reported $22 \%$ incidence of moulds with Aspergillus being predominantly isolated in their study (Ramani et al., 1993). The non dermatophyte moulds virtually damage the toenails especially which are damaged by disease or trauma (Andre $\mathbf{J}$ et al., 1987). In our study, we also found that all the non dermatophyte moulds were isolated from toenails. Shemer et al., (2009) suggested that where NDM infection is present in the first culture, the patient should be re-examined on next visit and three separate samples should be taken from the affected nail. If NDM is confirmed in all three of the cultures taken, the diagnosis of NDM is considered established and appropriate treatment can be started.

Although onychomycosis is not life threatening, it can cause a significant negative impact on the quality of life of infected patients. This study highlights that microbiological confirmation in case of onychomycosis is essential before the initiation of antifungal treatment.

\section{References}

Adhikari L, Gupta AD, Pal R, Sing TS. Clinico-etiological correlates of onychomycosis in Sikkim. Indian $\mathbf{J}$ Patthol Micribiol. 2009; 52: 194-7.

Andre J, Achten G. Onychomycosis. Int J Dermatol. 1987; 26: 481-490.

Banerjee U, Sethi M, Pasricha JS. Study of onychomycosis in India. Mycosis. 1989; 33 (7/8): 411-415.

Bokhari MA, Hussain I, Jahangir M et al., onychomycosis in Lahore, Pakistan. Int J Dermatol.1999; 38:591-595.

Chander Grover, Ananta Khurana. Onychomycosis: Newer insights in pathogenesis and diagnosis. Indian $\mathbf{J}$ Dermatology Venereology Leprology. 2012; 78:263-270

Ellis DH, Marley JE, Watson AB, Williams TG. Significance of non dermatophyte moulds and yeasts in onychomycosis. Journal of Dermatology. 1997; 194(1):40-42.

Greer DL. Evolving role of non dermatophytes in onychomycosis. Int $\mathbf{J}$ Dermatology. 1995; 34:521.

Gupta M, Sharma NL, Kanga AK, Mahajan VK, Tegta GR. Onychomycosis: clinicmycological study of 130 patients from Himachal Pradesh, India. Indian J Dermato Venereol Leprol. 2007; 76(6): 389-92.

Jorge Lopes, Alves SH. A ten years survey of onychomycosis in the central region of the Rio Grande do Sul, Brazil. Rev Inst Med Trolp Saov Paulo. 1999; 41:147149.

Kaur R, Kashyap B, Bhalla P. Onychomycisis- epidemiology, diagnosis and management. Indian $\mathbf{J}$ Medical Microbiology. 2008; 26: 10816.

Madhuri Jesudanam, G Raghu Ram Rao, D Joga Lakshmi, G Ratna Kumari. Onychomycosis: A significant medical problem. Ind $\mathbf{J}$ Dermatol Venerol Leprol. 2002; 68: 326-327.

Pradnya Veer, NS Patwardhan, AS Damle. Study of onychomycosis: prevailing 
fungi and pattern of infection. Indian $\mathrm{J}$ Medical Microbiology. 2007; 25:53-56t.

Puri DK, Sarin RC, Arora S. Pattern of dermatophytes affecting the nails. Ind J Dermatol Venerol Leprol. 1998; 44(2):91-94.

Ramani R, Srinivas CR, Ramani A, Kumari TG, Shivananda PG. Moulds in onychomycosis. Int J Dermato. 1993; 32:877-878.

Rigopuoulos D, Kotsiboulas V et al., Epidemiology of onychomycosis in southern Greece. Int J Dermatol. 1998; 35:s6-s7.

Shemer A, Davidovici B, Grunwald MH, Trau H, Amichai B. New criteria for the laboratory diagnosis of nondermatophyte molds in Onychomycosis. Br J Dermatol. 2009; 160:37-9.

Vinod S, Grover S, Dash K. A clinicomycological evaluation of onychomycosis. Indian J Dermatology Venereology Leprology. 2000; 66:238240.

\section{How to cite this article:}

Smita Kulkarni, Jayshree Bhakre and Damle, A.S. 2017. Clinico Mycological Study of Onychomycosis in Tertiary Care Centre of Maharashtra. Int.J.Curr.Microbiol.App.Sci. 6(11): 2718-2725. doi: https://doi.org/10.20546/ijcmas.2017.611.320 